\title{
Evaluación de la eficacia de cuatro dietas alimenticias sobre el crecimiento, desarrollo y producción de abono de la lombriz californiana (Eisenia foetida). ${ }^{27}$
}

\author{
Jessaily Gastón López y Marcos Miguel Martínez. ${ }^{28}$ \\ Enrique Cordón Suárez. ${ }^{29}$
}

\section{Resumen}

El presente trabajo se realizó en los campos de crianza y manejo de lombricultura de URACCAN, territorio comunal de Kamla, en donde se evaluó la eficacia de cuatro dietas alimenticias sobre el crecimiento, desarrollo y producción de lombricompuestos de la lombriz roja californiana Eisenia foetida. Los tratamientos evaluados fueron: estiércol de vaca (testigo), concentrado de $60 \%$ de hoja seca ${ }^{1}+40 \%$ estiércol de vaca, desperdicio de cocina, concentrado de $60 \%$ desperdicio cocina $+40 \%$ aserrín. Entre los principales resultados tenemos: que se registró una mayor tasa reproductiva en el tratamiento testigo (E. Vaca), seguido por el tratamiento Hoja seca + estiércol. De igual manera, de los cuatro tratamientos, los dos primeros brindan las condiciones óptimas para una producción de huevecillos. Se encontró una relatividad entre el número de muertos y el números de individuos, ya que a mayor población, mayor número de muertos. Del mismo modo, se encontró que en el tratamiento hoja seca + estiércol posee una alta razón de sobrevivencia, ya que por cada individuo muerto sobreviven 48. Se constató que la mayor cantidad de lombricompuestos se produce bajo alimentación de estiércol de vaca, seguido por el tratamiento hoja seca + estiércol de vaca.

Palabras Claves: Alimentación, crecimiento, desarrollo, producción, lombricompuestos.

\section{Introducción}

La lombricultura es una biotécnica que utiliza especies domésticas de lombriz, como una herramienta de trabajo, reciclando todo tipo de materia orgánica obteniendo

${ }^{27}$ Estudio realizado con el financiamiento del proyecto PATHWAY II "Support to Graduation Research Projects of Indigenous and Afro Descendant Students in URACCAN”, de la Fundación FORD.

28 Egresados de la Carrera de Ingienería Agroforestales de la Universidad de las Regiones Autónomas de la Costa Caribe Nicaragüense, Recinto Bilwi.

29 Coordinador de Ingeniería Agroforestal, Universidad de las Regiones Autónomas de la Costa Caribe Nicaragüense, Recinto Bilwi. Tutor de la investigación. 
como producto de este trabajo: humus, carne y harina de lombriz. Gutiérrez (www. compostadores.com. 2005).

\section{Clasificación Zoológica}

Reino : Animal

Tipo : : Anélido

Clase : Oligoqueto

Orden : Opistoporo

Familia : Lombricidea

Género : Eisenia

Especie : E. foetida

Las lombrices son hermafroditas incompletas, estas poseen dos sexos incompletos, la Eisenia foetida inicia su etapa de reproducción a los tres meses. Por lo tanto es necesario la cópula la cual ocurre cada 7 a 10 días. Luego cada individuo, coloca sus cápsulas (huevos en forma de pera amarillenta, de donde emergen de 2 a 21 lombrices después de un período de 14 a 21 días (Serebrinsky, 2004). El CIPAV (2006), revela a través de su estudio que la taza de profilicidad de la Eisenia foetida, es alta y esta puede llegar a aumentar el volumen de la lombrices hasta 300 veces por año y en algunos criaderos con un manejo excelente han llegado a aumentar hasta en 1,500 veces por año. Al nacer las lombrices son blancas, transcurridos 5 o 6 días se ponen rosadas y a los 120 días ya se parecen a las adultas siendo de color rojizo y estando en condiciones de aparearse (www.agriculturarticulos.com, 2001).

Gutiérrez (www.compostadores.com.2005) y M. Serebrinsky (2004), coinciden y mencionan que los alimentos orgánicos útiles para la alimentación pueden variar, destacándose entre ellos:

- Resto de aserrín e industrias relacionadas con la madera.

- Desperdicios de los mataderos.

- Residuos vegetales procedentes de la actividad agrícola.

- Estiércol de especies domésticas.

- Frutas y tubérculos.

- Fangos y basuras. 
$\operatorname{INTA}^{30}$ (2005), refiere que el suministro de los desperdicios de cocina a las lombrices, es una de las alternativas viables, fácil de obtener, asimilable por los individuos y para el productor, ya que este no tiene costo. La actividad de la lombricultura se puede realizar con diferentes métodos. Puede ser a través de cunas, crías en tolvas y cría en cajones, cada una de estas técnicas puede variar en lo más mínimo según el propósito del mismo, proporcionando excelentes resultados con un buen manejo (Jennyn, 2005). La humedad de los módulos bajo techo, se mantiene bajo dos estrategias: La primera se conoce la humedad del sustrato almacenado y suministrado, el cual es más fácil controlarlo en un rango de $70 \%$ a $80 \%$ de humedad. La segunda consiste en controlar las corrientes de aire y otros factores indeseables en las cunas.

De igual manera el INTA (2005) y Méndez (2004), coinciden en que la temperatura óptima para el crecimiento de la lombriz roja es de $15^{\circ}$ a $25^{\circ} \mathrm{C}$, en este rango se espera un máximo rendimiento de las lombrices, reproduciéndose de manera normal, alimentándose y produciendo lombricompuestos según el compost administrado. Las cunas deben de regarse con regularidad, teniendo en cuenta la época del año, generalmente en invierno se debe realizar cada 15 a 20 días, en verano dos veces por día. Es esencial que la humedad oscile entre el $70 \%$ a $80 \%$ y prever que la temperatura no supere los $32^{\circ} \mathrm{C}$, Jennyn (2005).

El lombricompuesto es un fertilizante orgánico, biorregulador y corrector del suelo cuya característica fundamental es la bioestabilidad, pues no provee lugar a la fermentación o putrefacción. El vermicompost contiene cuatro veces más nitrógeno, veinticinco veces más fósforo y dos veces y media más potasio que el mismo peso del estiércol de bovino, la producción de lombricompuesto de una lombriz adulta tiende a 0.60 gramos de humos aproximadamente, o sea el $60 \%$ del alimento consumido.

\section{Revisión de literatura}

\subsection{Lombricultura}

La lombricultura es una biotécnica que utiliza especies domésticas de lombriz, como una herramienta de trabajo, reciclando todo tipo de materia orgánica obteniendo como producto de este trabajo humus, carne y harina de lombriz. Gutiérrez (www. compostadores.com. 2005).

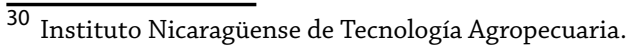




\subsection{Reproducción}

Las lombrices son hermafroditas incompletas, estas poseen dos sexos incompletos, la Eisenia foetida inicia su etapa de reproducción a los tres meses. Por lo tanto es necesario la cópula la cual ocurre cada 7 a 10 días. Luego cada individuo, coloca sus cápsulas (huevos en forma de pera amarillento). De donde emergen de 2 a 21 lombrices después de un período de 14 a 21 días (Serebrinsky, 2004).

\subsection{Crecimiento y desarrollo}

Después de la incubación de 2 a 21 días emergen las lombrices, las cuales tienen un color blanco y miden aproximadamente de 1 a $2 \mathrm{~mm}$ de longitud. A los 15 días las lombrices ya miden entre los 12 a 15 milímetros de longitud y tienen un color rosa pálido. Este ciclo de crecimiento y desarrollo se mantiene hasta que a los 90 días la lombriz ya es de color rojo oscuro y presenta un cóitelo, lo que indica que la lombriz ya tiene su tamaño definitivo de unos 8 a 10 centímetros y un peso de un gramo. Gutiérrez (www.compostadores.com.2005).

\subsection{Características de la especie}

La especie posee un cuerpo alargado, segmentado y con una simetría bilateral, tienen una longitud variable que oscila entre 2 a $15 \mathrm{~cm}$, con diámetro aproximado de $5 \mathrm{~mm}$ y peso de $0.8 \mathrm{gr}$. La Eisenia foetida posee un color rojizo del cual proviene su nombre (INTA 2005).

\subsection{Tratamiento y alimentación}

Gutiérrez (www.compostadores.com, 2005) y M. Serebrinsky (2004), coinciden y mencionan que los alimentos orgánicos útiles para la alimentación pueden variar, destacándose entre ellos:

- Resto de aserrín e industrias relacionadas con la madera.

- Desperdicios de los mataderos.

- Residuos vegetales procedentes de la actividad agrícola.

- Estiércol de especies domésticas.

- Frutas y tubérculos. 
- Fangos y basuras.

\subsection{Manejo y crianza}

El método de crianza en cuna es uno de los más efectivos y con más necesidad de cuido. Por lo que se tiene un horario de alimentación ya fijado, el cual se va desde la inseminación de las lombrices en la cuna, pasados 7 a 10 días se debe incorporar alimentos, seguido de cada semana que se le debe agregar de 3 a $4 \mathrm{~cm}$ de estiércol, Gutiérrez (www.compostadores.com, 2005).

\subsection{Lombricompuesto}

El INTA (2005), encontró que la producción de lombricompuesto en la instalaciones de crianza y manejo estará en dependencia del número de lombrices en existencia en el criadero, ya que se puede calcular esta a través de la multiplicación del número de lombrices por 0.6 gr por día por el tiempo de determinado.

Gutiérrez (www.compostadores.com, 2005), cita que en ocasiones pueden aparecer espécimen en los criaderos con lesiones e infecciones producidas por la acción de insectos o parásitos, la presencia de mosca, moskitos, ciempiés y hormigas. Si estas heridas son cerca del cóitelo la lombriz puede llegar a morir, por lo tanto se recomienda la revisión constante de las cunas y la limpieza manual de todo objeto o sustancia que pueda atraer organismos indeseados.

El mismo autor menciona, las bondades de la lombricultura como actividad productiva y correctiva del suelo en donde se enumeran algunas de ellas:

- Ayuda a producir abono orgánico, ecológicamente sano (humus).

- Método complementario para mejorar la rendición productiva en el campo.

- Brinda abono completo y efectivo, para mejorar el suelo.

- Este contiene hormonas y bacterias que estimulan en gran parte el desarrollo de las plantas.

- Funciona como un regenerador natural.

- Mejora las condiciones de vigor de las plantas y los vuelven más resistentes a las plagas y enfermedades. 
- La actividad lombricultural no necesita altos costos de inversión y mantenimiento.

- Equilibra la acidez o alcalinidad del suelo.

- Multiplica la eficiencia en la fertilización.

- Inactiva gran parte los residuos de los plaguicidas y químicos nocivos.

- Mejora la estructura y porosidad del suelo.

- Favorece la retención de la humedad.

\section{Materiales y método}

El presente trabajo se realizó en el área de crianza y manejo de lombricultura de la URACCAN, Recinto Universitario Kamla, Bilwi, RAAN, Nicaragua. Los datos de la estación meteorológica de esta zona de trabajo está ubicada bajo las condiciones climatológicas del trópico húmedo, con un promedio de $76 \%$ de humedad relativa, con una temperatura de $26{ }^{\circ} \mathrm{C}$ a $28^{\circ} \mathrm{C}$ y una precipitación anual de $3,000 \mathrm{~mm}$.

Cuadro No. 1. Variables a medir en el estudio

\begin{tabular}{|c|c|c|}
\hline Profilicidad & $\begin{array}{l}\text { Crecimiento y } \\
\text { desarrollo }\end{array}$ & $\begin{array}{c}\text { Producción } \\
\text { Lombricompuesto }\end{array}$ \\
\hline $\begin{array}{l}\text { Reproducción } \\
\text { Mortalidad } \\
\text { Huevecillos } \\
\text { R. Sobre vivencia }\end{array}$ & $\begin{array}{l}\text { Ind. }<5 \mathrm{~cm} \\
\text { Ind. } 6 \text { a } 10 \mathrm{~cm} \\
\text { Ind. > de } 10 \mathrm{~cm} \\
\text { Peso } \\
\text { Recesivo }\end{array}$ & Kg/Cuna \\
\hline
\end{tabular}

\section{1 Unidades experimentales}

Para albergar los tratamientos y las muestras se construyeron un total de 16 cajas de madera con dimensiones de $625 \mathrm{~cm}^{2}$, cada una de estas unidades estaban rotuladas con el fin de identificar y darle seguimiento según la descripción de las variables establecidas. 


\subsection{Muestras}

Como muestra de estudio, se seleccionó un total de 224 individuos en su etapa adulta, con una longitud mayor de $10 \mathrm{~cm}$ y un peso promedio de un $1 \mathrm{gr}$.

\subsection{Los tratamientos utilizados}

Se trabajó con un total de cuatro tratamientos, debido a sus características físicas y químicas pasaron por un proceso de precomposteo. La selección de estos tratamientos obedece a su gran abundancia y facilidad para obtenerlos en las comunidades y en las unidades productivas. Los sustratos corresponden a:

- Estiércol de vaca (testigo).

- Concentrado de $60 \%$ de hoja seca $+40 \%$ estiércol de vaca.

- Desperdicio de cocina.

- Concentrado de $60 \%$ desperdicio cocina $+40 \%$ aserrín.

\subsection{Variables a evaluar}

\subsubsection{Manejo del experimento}

El manejo del experimento se hizo de forma práctica, considerando el manejo por las personas comunitarias, la única variante que se hizo fue la manera ordenada en que se llevó el proceso para facilitar el levantado de información.

Se manejó una temperatura promedio de $27^{\circ} \mathrm{C}$ entre las cunas, de igual manera se mantuvo el $\mathrm{pH}$ de los tratamientos durante el proceso de precomposteo y después de su aplicación a los especímenes en estudio, se obtuvo un rango promedio de 6.8. En relación al control de la humedad, esta se logró manejar de forma sencilla basada en la irrigación de agua en las unidades experimentales, esta consistió en regarlas y a su vez ir realizando la prueba de puño, hasta el punto que esta lograse el valor óptimo en este caso de 70 a 80 por ciento.

Con respecto al control de plaga o de los enemigos de las lombrices se efectuó una vez a la semana, esta consistía en el retiro manual y ubicación de repelentes en la base de las cunas. Es importante mencionar que el control de los aspectos ambientales y el control de plagas se realizaron una vez a la semana, debido a la alta presencia de 
especímenes invasores y con el propósito de minimizar el efecto de la manipulación continua del experimento.

El levantamiento de datos del experimento se realizó cada 30 días de forma manual, contabilizando cada uno de los aspectos de las variables de estudios. La cosecha de abono y pesaje del mismo se realizó cada 30 días, se hizo de manera manual.

\subsubsection{Diseño experimental}

Con la finalidad de detectar diferencias estadísticas entre los tratamientos se estableció un diseño de bloque completo al azar (BCA), cuatro repeticiones. Fig. No. 1. A los resultados obtenidos, se les aplicó un análisis de varianza con un 95\% de confiabilidad y la separación de media a través de la prueba de Tukey.

\begin{tabular}{llll}
$\mathbf{I}$ & II & III & IV \\
\hline$T_{1}$ & $T_{2}$ & $T_{3}$ & $T_{4}$ \\
$T_{2}$ & $T_{3}$ & $T_{4}$ & $T_{1}$ \\
$T_{3}$ & $T_{4}$ & $T_{1}$ & $T_{2}$ \\
$T_{4}$ & $T_{1}$ & $T_{2}$ & $T_{3}$
\end{tabular}

Figura No.1. Distribución de los tratamientos

\section{Resultados y discusión}

A continuación se presentan los resultados obtenidos de la aplicación de los tratamientos descritos en la metodología, con sus respectivos análisis y discusiones.

\subsection{Profilicidad de lombriz roja californiana (Eisenia foetida)}

\subsubsection{Reproducción}

La reproducción es la variable que indica el número de individuos nuevos por cunas. Los resultados se presentaron en el sustrato testigo (estiércol de vaca) con 1079 individuos por caja; en segundo el sustrato hojas secas + estiércol con 483 individuos; en tercer lugar tenemos el tratamientos desperdicios de cocina con 123 individuos y por último desperdicios de cocina con 79 individuos por caja. 


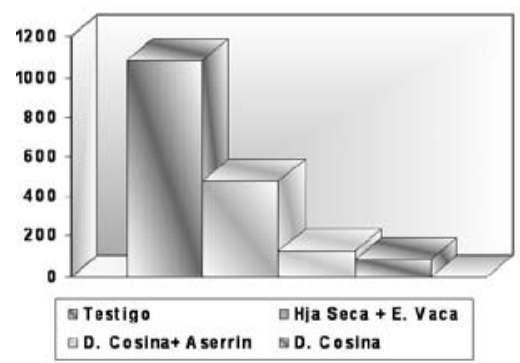

Figura No. 2. Comportamiento de número individuos por tratamientos

\subsubsection{Producción de huevecillos}

La mayor producción de huevecillos se presentó en el tratamiento testigo con 1,054 huevecillos y el tratamiento hoja seca + estiércol de vaca con una producción de 1,016 huevecillos, seguido por los tratamientos desperdicios de cocina + aserrín y desperdicios de cocina con 120 y 48 huevecillos respectivamente. La gráfica siguiente presenta los resultados.

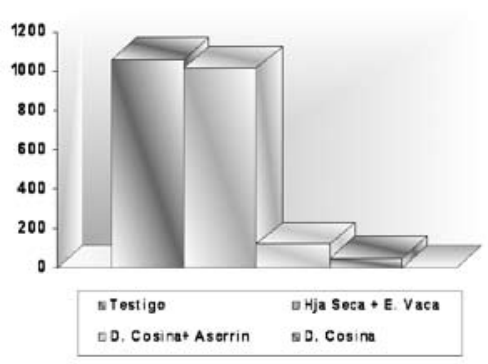

Figura No. 3 Comportamiento de producción de huevecillos por tratamientos

\subsubsection{Mortalidad}

Los mayores índices de mortalidad se presentaron en los sustratos testigo (estiércol de vaca) y desperdicios de cocina con 47 y 21 individuos muertos por cunas, seguido por los tratamientos desperdicios de cocina + aserrín y Hoja seca + estiércol de vaca con 19 y 18 individuos muertos por cunas. 


\subsubsection{Razón de sobrevivencia}

Con respecto a esta variable, se observa que por cada 21 individuos que sobreviven uno muere por causas diversas, siendo un resultado muy favorable en el experimento. A continuación la gráfica lo expresa.

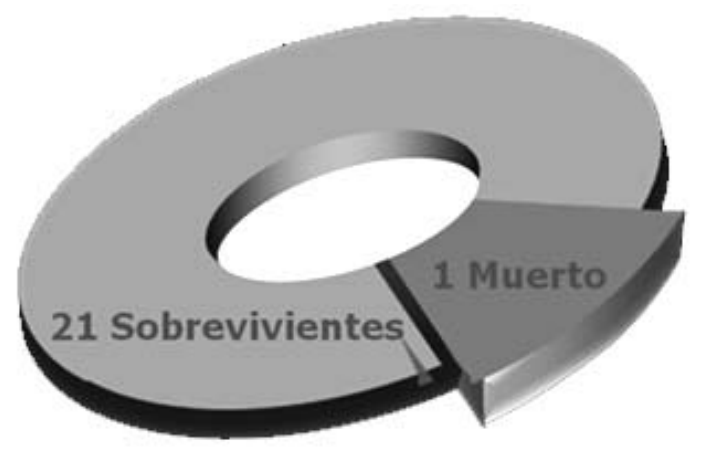

Figura No. 4. Comportamiento de sobrevivencia por tratamiento

De acuerdo con los tratamientos la sobrevivencia se presentó de la siguiente manera: una mayor sobrevivencia en el tratamiento hoja seca + estiércol de vaca con una razón de 48 individuos por cada uno que muere por cualquier causa, seguido por tratamiento testigo con 30 individuos sobrevivientes por cada uno que muere, finalmente los demás tratamientos.

\subsection{Crecimiento y desarrollo de la lombriz roja californiana}

\subsubsection{Individuos con talla $<5 \mathrm{Cm}$ de longitud}

Es importante conocer la talla de las lombrices, porque este nos ayuda a estimar la cantidad de eclosiones de individuos y determinar los niveles de crecimientos para la segunda etapa. A continuación los resultados del ensayo se muestran en el siguiente gráfico. 


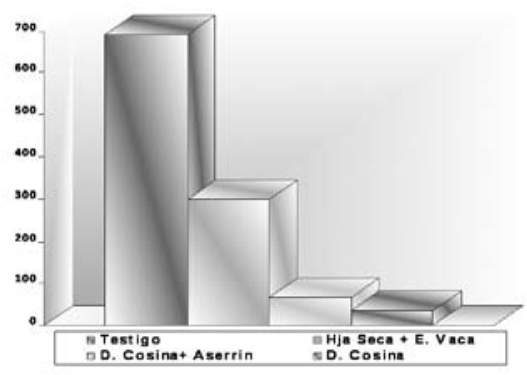

Figura No. 5. Individuos con talla $<$ de $5 \mathrm{~cm}$ de longitud por tratamientos.

Se encontró que en el tratamiento testigo se encontraron un mayor número de individuos con total de 692.25 especímenes; en segundo lugar el tratamiento Hoja seca + estiércol con 300.5 individuos y finalmente los tratamientos desperdicios de cocina + aserrín y desperdicio de cocina con 66.75 y 34.75 individuos respectivamente.

\subsubsection{Individuos con talla de 6 a $10 \mathrm{~cm}$ de longitud}

La talla de 6 a $10 \mathrm{~cm}$ es un indicador del número de individuos que se encuentran en la etapa de reproducción.

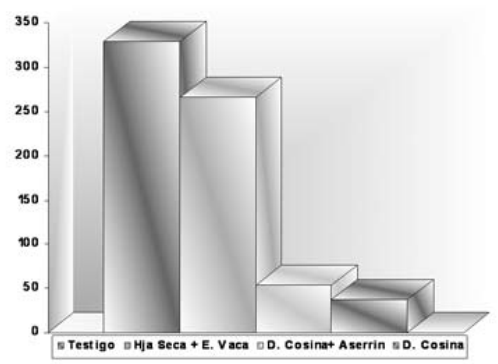

Figura No. 6. Individuos con talla de 6 a $10 \mathrm{~cm}$ longitud por tratamientos

Los resultados del ensayo presenta la una mayor permanencia de individuos de la talla de 6 a $10 \mathrm{~cm}$ de longitud con un total de 329 individuos por cajas; en segundo lugar tenemos el tratamiento Hoja Seca + estiércol con 265 individuos y finalmente desperdicios de cocina + aserrín con 54.25 y finalmente desperdicio de cocina 37 individuos. 


\subsubsection{Individuos con talla mayor $\mathrm{de} 10 \mathrm{~cm}$ de longitud}

La talla mayor de $10 \mathrm{~cm}$ es un indicador del número de individuos que encuentra en la etapa adulta de su ciclo de vida, individuos totalmente desarrollados. Los resultados muestran un mayor número de individuos en el sustrato testigo con 58.25 y en segundo lugar tenemos el tratamiento hoja seca + estiércol de vaca con 23.25 individuos por cunas y por último tenemos los tratamientos desperdicios de cocina + aserrín con 7.75 y desperdicios de cocinas 7.75 individuos.

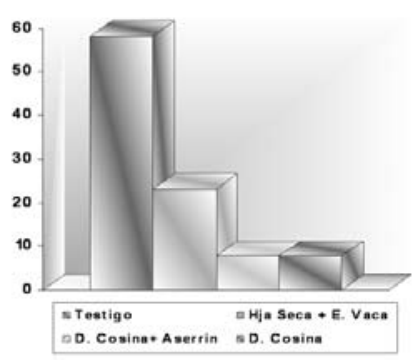

Figura No. 7. Individuos con talla de mayor de $10 \mathrm{~cm}$ longitud por tratamientos

\subsubsection{Peso de individuos adultos}

El pesos de los individuos, es un indicador que demuestra el grado de buen desarrollo alcanzado por los individuos, se relaciona con individuos sanos, resistentes a los eventos adversos en su entorno y individuos seleccionados para pie de cría para nuevas generaciones. En este estudio los resultados demuestran que el promedio de peso de los individuos adultos es de $1.1 \mathrm{gr}$.

\subsection{Comportamiento de recesión en crecimiento}

Los resultados obtenidos según análisis de datos relacionados a crecimiento y desarrollo de los especímenes, se encontró en los tratamientos testigo, hoja seca + estiércol, tienen un alto porcentaje de recesión en la etapa de crecimiento de los individuos de menor de $5 \mathrm{~cm}$ de talla. A continuación se muestra comportamiento recesivo durante el ensayo. 


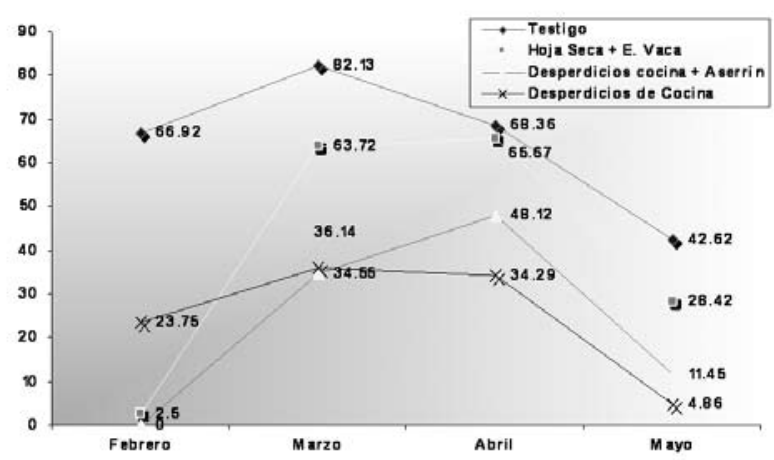

Figura No. 8. Comportamiento recesivo de los especímenes durante el ensayo por tratamientos

\subsection{Producción de Lombricompuesto}

La mayor producción se dio en los sustratos testigo de $5.72 \mathrm{~kg}$ por caja; en segundo lugar tenemos el tratamiento Hoja seca + estiércol de vaca con $3.47 \mathrm{~kg}$ y finalmente tenemos los tratamientos desperdicios de cocinas y desperdicios de cocina + aserrín con $1.9 \mathrm{~kg}$ y $1.5 \mathrm{~kg}$.

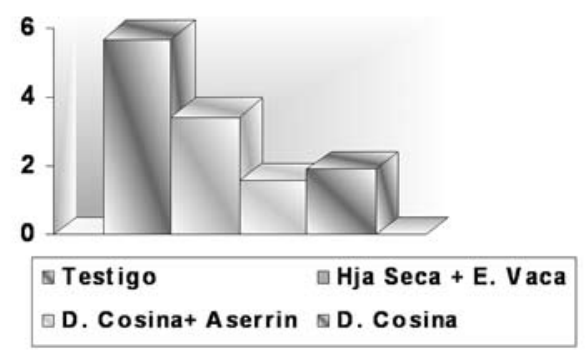

Figura. No. 9. Comparación de producción de lombricompuestos por tratamientos

\subsection{Presencia de plagas}

Durante el desarrollo del ensayo, se encontró alta presencia de organismos invasores principalmente en los tratamientos desperdicios de cocina + aserrín, encontrándose con mayor frecuencia insectos como escarabajos (Agamopus lampros), Zompopos (Atta cephalotes), la moscas (Linnaeus) y cien pies (Lithobius sp). Mientras que en el 
tratamiento desperdicios de cocina, los organismos no deseados fueron Zompopos (Atta cephalotes), la moscas (Linnaeus) y cucarachas (Peri planeta americana)

\section{Conclusiones}

El comportamiento de la lombriz roja californiana (Eisenia foetida) se basó en su capacidad de reproducción. Es así que se registró una mayor tasa reproductiva en el tratamiento testigo (E. Vaca), seguido por el tratamiento hoja seca + estiércol.

De los cuatro tratamientos suministrados, se determinó que el mayor número de muerte de individuos se presentaron en el tratamiento testigo (E. Vaca) y hoja seca + estiércol.

Encontrándose de esta manera evidencia significativa de relatividad entre número de muertos y números de individuos. A mayor población, mayor números de muertos.

En el tratamiento hoja seca + estiércol, por cada individuo muerto sobreviven un total de 48 individuos, siendo esta una alta razón de sobrevivencia. Hallando relatividad significativa entre porcentaje de recesión y número de individuos, por lo que a mayor población alto porcentaje de recesión en los individuos.

La mayor cantidad de lombricompuestos producido se da en el tratamiento testigo (E. Vaca), seguido por el tratamiento hoja seca + estiércol de vaca.

\section{Recomendaciones}

Se recomienda el uso de estiércol de vaca o bien el concentrado de hoja seca + estiércol como materia prima principal en la alimentación de la lombriz Roja Californiana (Eisenia foetida) para la cría de lombrices.

Se sugiere la utilizar de la mezcla Hoja seca + estiércol de vaca en las cajas alberges, con el objetivo de aprovechar las condiciones de ambiente que brinda este sustrato.

Se recomienda, evacuar las lombrices cuando la población rebasa los 350 individuos adulto por cada 50 centímetro cuadrado, ya que la sobrepoblación induce a la muerte. Por tanto se debe llevar un registro de la reproducción. 
Al no encontrarse suficientes estudios en Nicaragua y en nuestras Regiones Autónomas, se aconseja el seguimiento y profundización del tema.

Realizar estudios específicos sobre las propiedades física y químicas del lombricompuesto.

Promover la lombricultura en nuestras comunidades indígena, en donde esta biotecnología complemente las actividades productivas tradicionales.

\section{Referencias bibliográficas}

Serebrinsky, M. (2004). Comportamiento reproductivo de la Eisenia foetida según estación del año y tipo de alimentación. Colombia.

CIPAV, (2006). Biología de la "Eisenia foetida" en Cautiverio.

INTA (2005). Estudio de influencia del tipo de estiércol en la transformación de la materia orgánica tratada mediante proceso de comportado y vermicompostado. Argentina.

Jennyn, (2005). Manual Básico de Lombricultura para Condiciones Tropicales. Estela, Nic. Pp 37.

Méndez, J. (2004). Comportamiento reproductivo de la lombriz roja californiana. Argentina

http://www.compostadores.com, (con acceso mes de noviembre 2007) 